\title{
Experimental evidence of single-stranded DNA adsorption on multi-walled carbon nanotubes
}

\author{
Franco Tardani, ${ }^{\dagger}$ Stefano Sarti, $\ddagger$ Simona Sennato, ${ }^{*}, \dagger, \ddagger$ Manuela Leo,, Patrizia \\ Filetici, " Stefano Casciardi,§ Pier Giorgio Schiavi, $\|$ and Federico Bordi ${ }^{\ddagger} \dagger$ \\ †Istituto dei Sistemi Complessi (ISC)- CNR, UOS Roma Sapienza - P.zzle A. Moro, 2 - \\ 00185 Roma - Italy
}

tDipartimento di Fisica - Sapienza Università di Roma - P.zzle A. Moro, 2 - 00185 Roma Italy

IIstituto di Biologia e Patologia Molecolari-CNR, Sapienza Università di Roma, Piazzale A. Moro 5, 00185 Roma, Italy

§Istituto Nazionale per l'Assicurazione contro gli Infortuni sul Lavoro, Dipartimento di medicina, epidemiologia, igiene del lavoro e ambientale Via Fontana Candida 1, 00078 Monte Porzio Catone (RM), Italy

||Dipartimento di Chimica - Sapienza Università di Roma - P.zzle A. Moro, 5 - 00185

$$
\text { Roma - Italy }
$$

E-mail: simona.sennato@roma1.infn.it

Phone: +3906 49913593 


\section{SUPPLEMENTARY INFORMATION}

\section{Atomic Absorption Spectroscopy for Analysis of Potas- sium}

The degree of substitution of potassium in the Polyadenylic acid potassium salt (PolyA) was evaluated by flame atomic absorption spectroscopy (Analytic Jena ContrAA300). The analysis was carried out using an atomizer with an air/acetylene burner. The wavelength used for the determination of potassium was $766 \mathrm{~nm}$. A calibration curve with a concentration range between 0.5-5 $\mathrm{ggml}^{-1}$ was obtained by analyzing four different samples prepared by an appropriate dilution the stock solution (Merck). Potassium determination in PolyA has been performed dissolving a known amount of sample in MilliQ Ultrapure grade water and the resulting solution was diluted in order to have a final concentration within the calibration range.

\section{Procedure for Subtraction of the Electrode Polarization Contribution to Dielectric Spectra}

The contribution of electrode polarization has been modeled as a Constant Phase Angle (CPA) element, $K(i \omega)^{\alpha}$, where $\omega=2 \pi \nu$ is the angular frequency of the electric field, in

series with the sample impedance. ${ }^{1}$ Writing the measured impedance $Z_{m}$ as $Z_{m}=\left(i \omega C_{0} \tilde{\epsilon}_{m}\right)^{-1}$, where $C_{0}$ is the capacitance of the empty cell, one has

$$
\frac{1}{i \omega C_{0} \tilde{\epsilon}_{m}}=\frac{1}{K(i \omega)^{\alpha}}+\frac{1}{i \omega C_{0} \tilde{\epsilon}_{s}}
$$

where $\tilde{\epsilon}_{s}$ is the true permittivity of the sample and hence 


$$
\frac{C_{0}}{K}(i \omega)^{1-\alpha}=\frac{1}{\tilde{\epsilon}_{m}}-\frac{1}{\tilde{\epsilon}_{s}}
$$

The phenomenological parameters $K$ and $0 \leqslant \alpha \leqslant 1$ depend on the nature and roughness of the electrodes and on sample conductivity ${ }^{1}$ and have been determined by an iterative fitting procedure. Briefly, a tentative $\tilde{\epsilon}_{s}$ curve with a relaxation in the $10^{4} \mathrm{~Hz}$ range has been calculated and used to obtain $\frac{1}{\tilde{\epsilon}_{m}}-\frac{1}{\tilde{\epsilon}_{s}} \sim(i \omega)^{1-\alpha}$ in an as wide as possible frequency range. Once $\alpha$ is known, the parameter $C_{0} / K$ has been adjusted to fit the data at low frequencies. Once $C_{0} / K$ and $\alpha$ are determined in this way, the true sample permittivity is determined as

$$
\frac{1}{\tilde{\epsilon}_{s}}=\frac{1}{\tilde{\epsilon}_{m}}-\frac{C_{0}}{K}(i \omega)^{1-\alpha}
$$

This procedure allowed us to extend the frequency range of the spectra down to slightly more than $1 \mathrm{kHz}$. Once the electrode polarization contribution is subtracted, we also subtract the contribution of water, obtained from literature data. ${ }^{2}$ The relaxations due to the DNA and the DNA-MWCNT complexes fall in the $\mathrm{MHz}$ range $(1 \mathrm{MHz} \leq \nu \leq 1 \mathrm{GHz})$. However, the availability of a sufficiently wide low frequency tail allows for a more accurate determination of the parameters defining these relaxations. The quantitative analysis of the parameters obtained from the fit of the lower tail of the spectra $\left(\nu<10^{4} \mathrm{~Hz}\right)$ is out from the scope of this investigation.

\section{Determination of Free Counterion Fraction $\theta$}

According to the model of Ito et al, ${ }^{3-5}$ the polarizability of each free counterion (i.e. counterion not condensed along the polyelectrolyte chain, in the meaning of Manning's theory ${ }^{3,6,7}$ ), $\alpha_{i o n}$, is determined by the square of its effective dipole, i.e. its charge $e$ (for univalent coun- 
terions, as in our case) times the polarization distance $L$, divided by the thermal energy ${ }^{3,8,9}$

$$
\alpha_{i o n} \approx \frac{(e L)^{2}}{k T}
$$

Then, the dielectric increment $\Delta \varepsilon$ is simply the product of the number density of free counterions $\theta c$ and their polarizability

$$
\Delta \varepsilon \approx \theta c \alpha_{i o n} \approx \theta c \frac{(e L)^{2}}{k T}
$$

By geometrical considerations, a scaling dependence of $\Delta \varepsilon$ on the concentration with exponents $1 / 3$ for the dilute and 0 (constant) for the semidilute regime can be obtained ${ }^{3}$

$$
\Delta \varepsilon \sim C_{D N A}^{\gamma}
$$

By combining eq. 1 and the relation $\tau \approx \frac{L^{2}}{6 D}$ an estimate of the fraction $\theta$ of free counterions can be easily calculated $\mathrm{as}^{3}$

$$
\theta \approx \frac{\Delta \varepsilon}{c \alpha_{i o n}} \approx \frac{\Delta \varepsilon k T}{c(e L)^{2}} \approx \frac{\Delta \varepsilon k T}{c e^{2} 6 D \tau}
$$

or, in term of the Bjerrum length $l_{B}=\left(e^{2}\right) /\left(4 \pi \varepsilon_{0} \varepsilon_{w} k T\right)$

$$
\theta \approx \frac{\Delta \varepsilon}{c 6 D \tau l_{B} \varepsilon_{w}}
$$

\section{References}

(1) Bordi, F.; Cametti, C.; Gili, T. Reduction of the contribution of electrode polarization effects in the radiowave dielectric measurements of highly conductive biological cell suspensions. Bioelectrochemistry 2001, 54, 53-61. 
(2) Ellison, W.; Lamkaouchi, K.; Moreau, J.-M. Water: a dielectric reference. J. Mol. Liq. 1996, $68,171-279$.

(3) Bordi, F.; Cametti, C.; Colby, R. H. Dielectric spectroscopy and conductivity of polyelectrolyte solutions. J. Phys.: Condens. Matter 2004, 16, R1423-R1463.

(4) Tomić, S.; Babić, S. D.; Vuletić, T.; Krča, S.; Ivanković, D.; Griparić, L.; Podgornik, R. Dielectric relaxation of DNA aqueous solutions. Phys. Rev. E 2007, 75, 021905.

(5) Ito, K.; Yagi, A.; Ookubo, N.; Hayakawa, R. Crossover behavior in high-frequency dielectric relaxation of linear polyions in dilute and semidilute solutions. Macromolecules 1990, 23, 857-862.

(6) Manning, G. The molecular theory of polyelectrolyte solutions with applications to the electrostatic properties of polynucleotides. Q. Rev. Biophys. 1978, 11, 179-246.

(7) Manning, G. Electrostatic free energy of the DNA double helix in counterion condensation theory. Biophys. Chem. 2002, 101-102, 461-473.

(8) Bordi, F.; Colby, R. H.; Cametti, C.; De Lorenzo, L.; Gili, T. Electrical conductivity of polyelectrolyte solutions in the semidilute and concentrated regime: the role of counterion condensation. J. Phys. Chem B 2002, 106, 6887-6893.

(9) Bordi, F.; Cametti, C.; Gili, T.; Colby, R. H. Dielectric relaxations in aqueous polyelectrolyte solutions: a scaling approach and the role of the solvent quality parameter. Langmuir 2002, 18, 6404-6409. 